\title{
Generating a Variable Uniform Magnetic Field Suitable for Fatigue Testing Magnetorheological Elastomers Using the Bubble Inflation Method.
}

\author{
Dave Gorman \\ Technological University Dublin, david.gorman@tudublin.ie \\ Stephen Jerrams \\ Technological University Dublin, stephen.jerrams@tudublin.ie \\ Ray Ekins \\ Technological University Dublin, ray.ekins@tudublin.ie
}

See next page for additional authors

Follow this and additional works at: https://arrow.tudublin.ie/archastrocon

Part of the Architectural History and Criticism Commons

\section{Recommended Citation}

Gorman, D., S. Jerrams, et al. (2013). Generating a variable uniform magnetic field suitable for fatigue testing magnetorheological elastomers using the bubble inflation method. ,i>Constitutive Models for Rubber VIII, CRC Press: 671-675.

This Conference Paper is brought to you for free and open access by the Archaeoastronomy Research at ARROW@TU Dublin. It has been accepted for inclusion in Conference Papers by an authorized administrator of ARROW@TU Dublin. For more information, please contact arrow.admin@tudublin.ie, aisling.coyne@tudublin.ie,gerard.connolly@tudublin.ie.

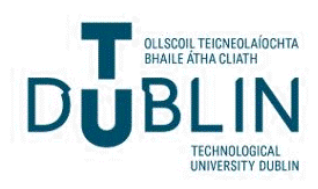




\section{Authors}

Dave Gorman, Stephen Jerrams, Ray Ekins, and Niall Murphy

This conference paper is available at ARROW@TU Dublin: https://arrow.tudublin.ie/archastrocon/4 


\title{
Generating a variable uniform magnetic field suitable for fatigue testing magnetorheological elastomers using the bubble inflation method.
}

\author{
D. Gorman, S. Jerrams, R. Ekins, \& N. Murphy \\ Centre for Elastomer Research, Dublin Institute Technology, Republic of Ireland
}

\begin{abstract}
This paper investigates the magnetic field generated by an electromagnetic array and whether it is suitable for the physical testing of magnetorheological elastomers (MREs) using bubble inflation. This will be achieved by comparing the magnetic field generated by the array with the simulated field calculated by an FEA model. The array will be evaluated with detailed measurements of the magnetic flux strength and direction over the entire sample volume. The magnetic flux versus the time the array is powered in order to ensure it is capable of providing a reliable magnetic field for the duration of a biaxial fatigue test of an MRE.
\end{abstract}

\section{INTRODUCTION}

An MRE is a smart elastomer which undergoes a change in its physical properties (particularly an increase in modulus) when an external magnetic field is applied to it (Boczkowska and Awietjan, 2009). The changes in physical properties of the MRE occur due to ferromagnetic particles, added to the elastomer matrix during the curing process, which align with the applied magnetic field. The applied field results in dipole dipole interactions between the particles which move to screen each other from the field and adopt a minimum energy configuration (Stepanov et al., 2007). There are two types of MREs independent of the elastomer matrix or ferromagnetic particles used. These are isotropic and anisotropic MREs (Varga et al., 2006). For isotropic MREs no magnetic field is applied to the mix during the curing process resulting in an almost homogeneous distribution of the ferromagnetic particles, while for anisotropic MREs an external magnetic field is applied to the mix during the curing process resulting in the formation of aligned particle chains. Once the matrix has hardened, the particle mobility is reduced and the aligned chains remain in position. MREs with aligned particles normally exhibit a greater magnetorheological effect than isotropic MREs

To date MRE testing has predominantly been carried out on uniaxially loaded samples with the magnetic field stated as uniform in both flux density and direction over the entire sample volume. However such a specification is of questionable value due to the paucity of information about the magnetic field applied during testing (Gorman et al., 2012). Irres- pective of the magnetic field applied, a magnetic flux density has a specific maximum value at a point and this value will fall away with movement away from that point in any direction. Hence if it is stated that a test on a sample of MRE is performed in a magnetic field of, for example, $400 \mathrm{mT}$, this provides no information about the depth of that field or the change in flux density in mutually perpendicular planes.

As bubble inflation tests used for biaxial testing take place over a larger volume than standard uniaxial testing on dumbbell shaped samples (Murphy et al., 2011), a more complex magnetic array is required (Gorman et al., 2011).

A magnetic field of uniform strength is required as the MR (magnetorheological) effect is due to the particles attempting to align in the direction of the applied magnetic field and the alignment is caused by magnetic dipole interactions. The behavior of a component subject to displacement would be difficult to predict or control if the flux density varied greatly over the displacement volume of the component. As the force which causes the particle alignment is dependent on the flux density, the MR effect is dependent on both the direction and flux density of an applied magnetic field. Application of a non uniform field would result in different effects at points over the sample volume. The force on a magnetic dipole $(\mathrm{m})$ in a magnetic field $(\mathrm{B})$ is given by

$$
F=\nabla(m . B)(\text { Grant and Phillips, 1975). }
$$

A uniform field direction is important for both uniaxial and biaxial testing of MRE samples as the direction of the field force and relative alignment be- 
tween particles result in varying changes in mechanical properties. It has been reported by (Varga et al., 2006) and (Boczkowska and Awietjan, 2009) that when the magnetic field is applied parallel to the particle chains it produces a greater MR effect than the same flux density applied perpendicular to the particle chains.

\section{MODELLING THE MAGNETIC FIELD}

\subsection{Uniform magnetic fields}

A Halbach cylinder is an array of permanent magnets arranged in a cylinder which produces a uniform magnetic field, in one plane and over acceptable limits.

A model with four electromagnets has been proposed based on the geometry of an open access Halbach cylinder used by (Hills et al., 2005). The proposed model is shown Figure 1

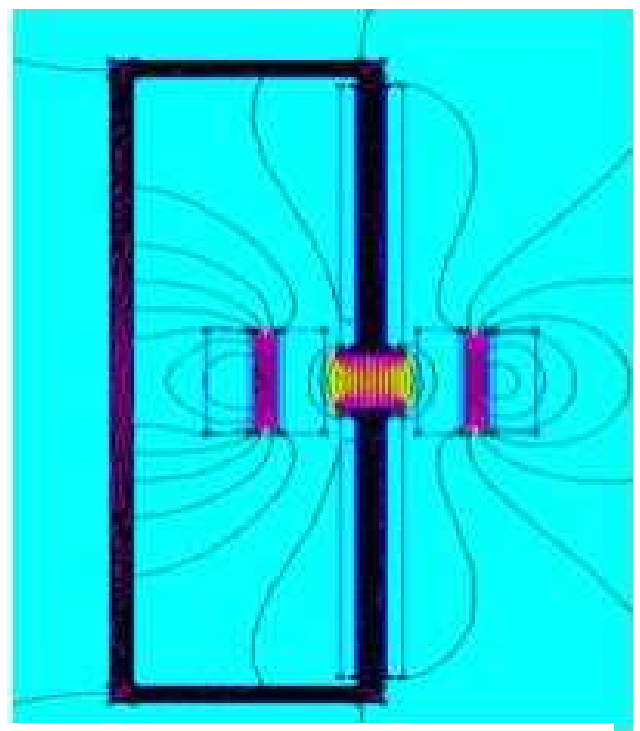

Figure 1 Proposed electromagnetic array

The FEA model calculates the simulated field and typical results are shown in Figure 2 for the field operating between the poles of the central coils.

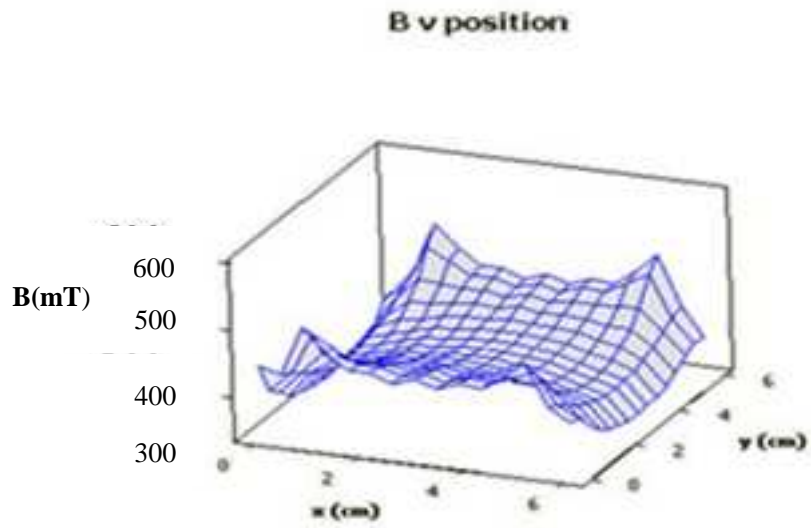

Figure 2 depicts that the simulated field has both a relatively uniform flux density of over $400 \mathrm{mT}$ and is of uniform direction. Further details of the modelling process were presented in previous work. (Gorman et al., 2011).

\section{MANUFACTURING A PROTOTYPE OF THE ELECTROMAGNETIC ARRAY}

\subsection{Materials used}

The electromagnetic array presented in Figure 1 consists of 4 main parts; the endplates, cores, windings and the magnetic circuit. Both the cores and the magnetic circuit are made from $99.9 \%$ pure soft magnetic iron of $19.1 \mathrm{~mm}$ diameter. The windings are $1 \mathrm{~mm}$ diameter copper wire with an insulation coating. The only endplates shown in the simulated model are those for the central coils which are constructed using magnetic steel.

The other endplates for the central coils and those of the side coils are made of an aluminum alloy. These endplates are not included in the simulated model as aluminum alloys have a relative permeability of $1\left(\mu_{\mathrm{r}}=1\right)$ and do not effect the magnetic field.

\subsection{The cooling system}

The main drawback of using an electromagnetic array over a fixed permanent magnet array is it requires constant input of energy in the form of an electric current to maintain the magnetic field. This results in increased temperatures and as the temperature rises the resistance of the array increases resulting in a drop of magnetic field strength. If an electromagnetic array is to be used for evaluating the fatigue life of any MRE, it is important that the magnetic field is constant over the test lifetime.

The overcome the problems associated with heat buildup the electromagnetic array was water cooled. To facilitate the cooling system, the cores were covered by a perforated copper sleeve and the windings were placed on this sleeve. This provided a space for cold water to enter the coils between the core and the windings. The water then flowed through radial channels between the windings to the outside of the coil. The channels were produced by introducing corrugations in the wire as it was being wound. This arrangement is shown in Figure 3. Figure 3a shows the windings on the completed electromagnets where as Figure $3 b$ shows the corrugations in the wires 


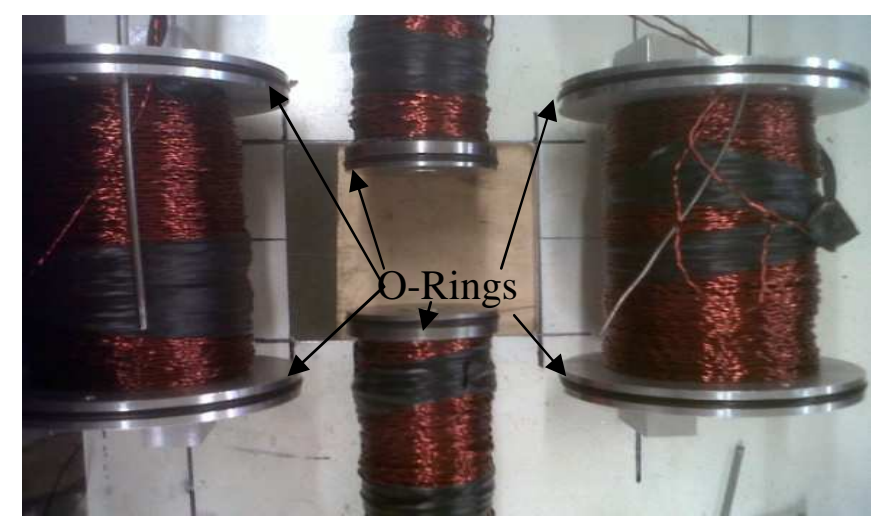

Figure 3a Coils wound with corrugated wire

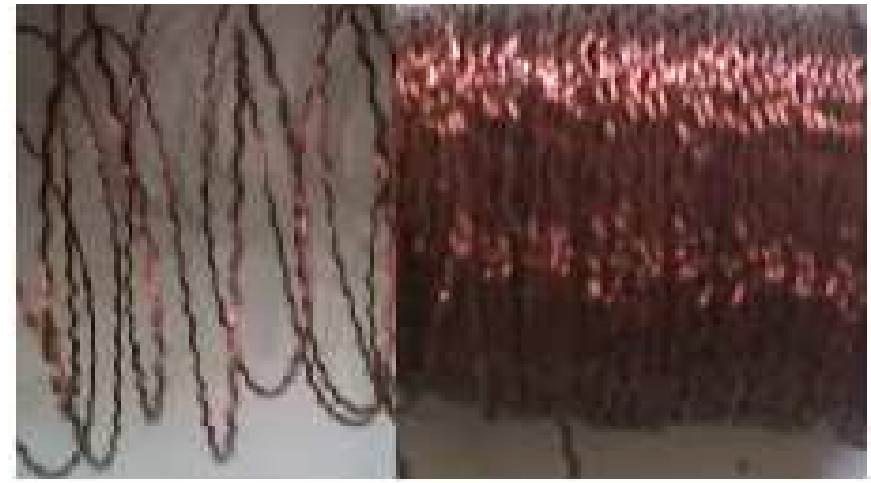

Figure $3 \mathrm{~b}$ Corrugated coils for cooling purposes

The cooling system design was also incorporated into the endplates of the electromagnet. The water both enters and exits the electromagnets through the endplates and they form a watertight seal with the casings. This is achieved by introducing an annular grove and a recess in the endplate. The annular groove is for an O-ring (shown in Figure 3a) which provides a water tight seal with the casing and the recess holds the copper sleeve which allows cool water to enter between the core and the winding.

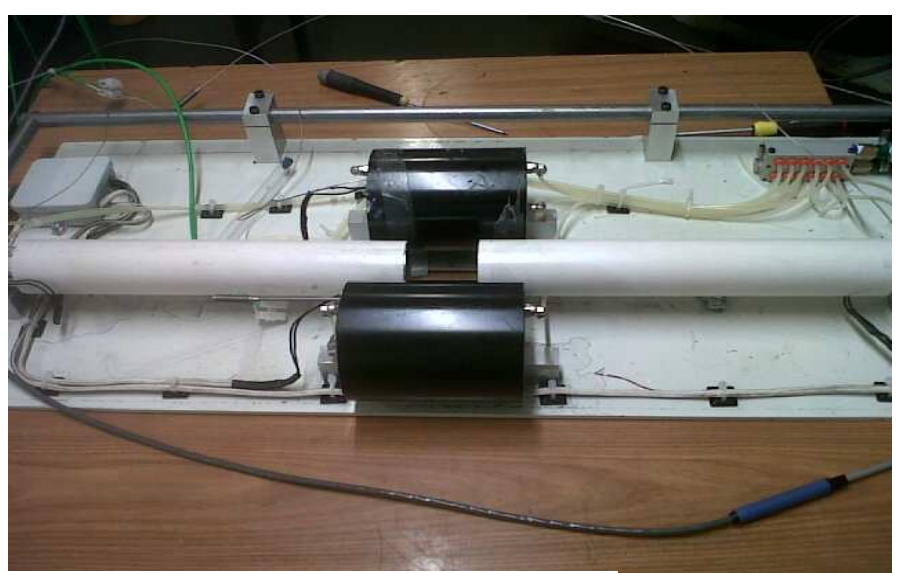

Figure 4 Assembled array

Figure 4 shows the assembled array with the electromagnets fully encased in their cooling sleeves which unsure the array is watertight and thermocouples attached to each electromagnet.

\section{RESULTS}

\subsection{Field map}

The results presented in this section are those measured directly with a 3 channel Hall probe and Gauss meter for the array subject to a current of 15amps dc through each coil. The results for this current are shown in Figure 5. The temperature of the array was maintained relatively constant which each coil reaching a steady temperature of between $28^{\circ} \mathrm{C}$ and $33^{\circ} \mathrm{C}$, ensuring there was virtually no change in flux density due to a change in resistance.

\section{B versus position}

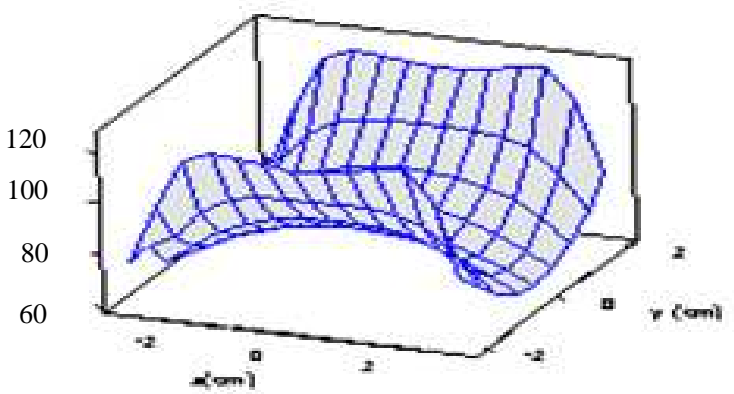

Figure 5 Flux density versus position

\subsection{Comparing mapped flux density for the prototype with the model}

By comparing the graphs in Figures 2 and 5 it is clear that the mapping and the model do not agree. The largest difference between the two is that the flux density reached $400 \mathrm{mT}$ in the simulation but was recorded at between $120 \mathrm{mT}$ and $80 \mathrm{mT}$ during the field mapping process.

This low flux density by comparison with computer models was caused by two factors: Firstly the magnetic circuit reached a local saturation causing the flux density (B) in the iron to level off as the magnetic field $(\mathrm{H})$ was increased. In the prototype, this resulted in the flux density plateauing as current increased. Figure 6 shows current in the coils versus the flux density at the centre point between the poles (the failure position of the bubble inflation sample). 


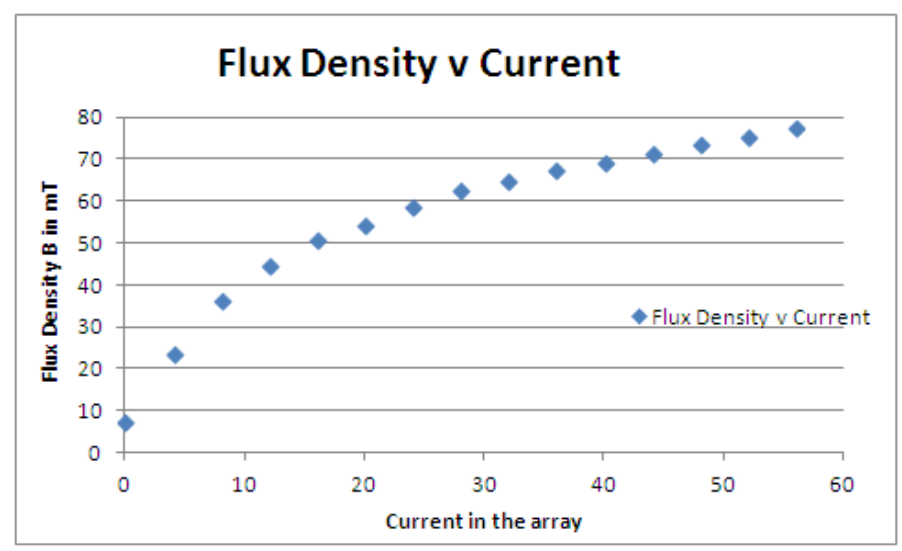

Figure 6 Flux Density versus Current in array

A possible cause of lower saturation in the prototype is due to the introduction of low carbon steel screws and air pockets into the circuit. These where introduced into the model and are shown in Figure 8

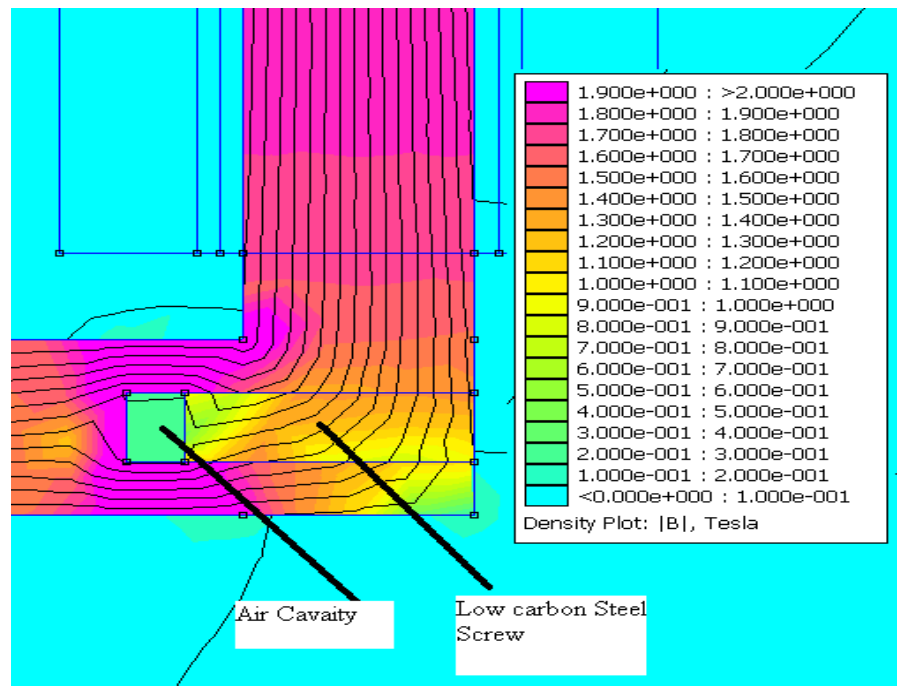

Figure 7 Model with low carbon steel and air pockets

It can be seen from Figure 7 that the local field is distorted by the air gaps and the change in material properties due to the presence of the screws. The points above and below the air gap reach a saturation point of around 2 Tesla. This results in a drop in the field across the air gap but does not account for the total fall. This situation can be seen in Figure 8

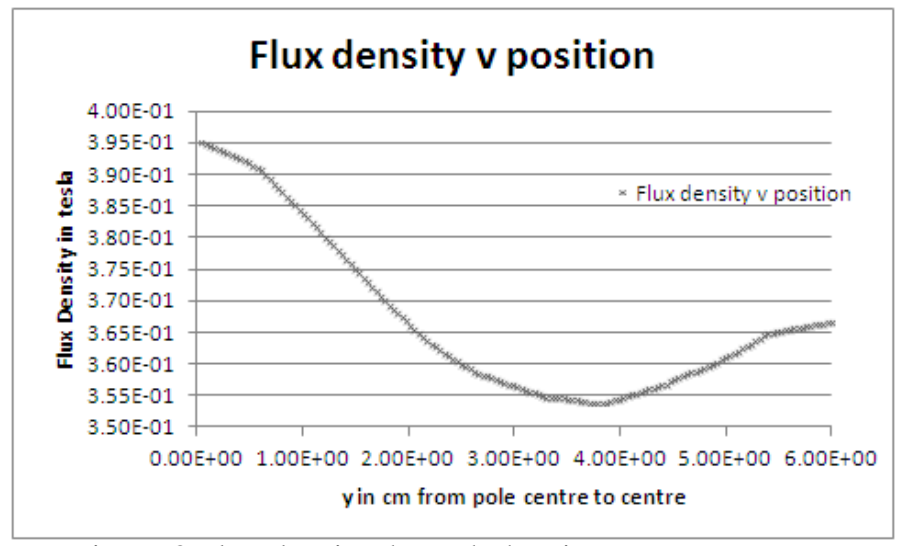

Figure 8 Flux density through the air gap
The second cause of a lower than predicted measured flux density is due to the flux being spread over a large volume inevitably causing a difference between the 2D model and the 3D array. The pole pieces have a large surface area that will not be modelled in the 2D simulation and in reality the flux density can loop back providing more return pathways.

Despite the prototype having a the lower flux density than was simulated in the model, the mapping and model produced a similar field profile in terms of uniformity of direction and flux density. It was observed almost all of the total field (99\%+) travelled in the principal y direction (from pole to pole) in the centre and just over $87 \%$ at the edge regions $(\mathrm{x}-3 \mathrm{~cm}$ and $3 \mathrm{~cm})$.

\section{CONCLUSIONS}

The array shown in figure 5 and mapped in figure 6 is capable of producing a magnetic field having the required uniformity of direction and flux density to allow for the testing of MREs using bubble inflation. However until the problems with early saturation of the magnetic circuit and the drop in flux density due to the large surface areas of the pole pieces have been overcome, a full test program over a practical range of fields for MREs cannot be employed for biaxial dynamic testing.

The difference between the mapped and simulated field highlights the need for detailed 3D mapping of any magnetic field used to test an MRE sample. Both modelling and testing show that single value field strength is inadequate when testing samples of even a few $\mathrm{cm}^{3}$ in volume.

It has also been shown that an electromagnetic array can be maintained at a constant temperature/resistance for the duration of a fatigue test without field drop off.

\section{PROPOSED FURTHER WORK}

The magnetic array will be evaluated over a greater range of currents and volumes to provide a map with a greater resolution and depth. Also the pole pieces will be modified to reduce the difference between the surface area of the magnetic material in the pole pieces and the cross-sectional area of the electromagnets cores. It is expected that this will cause the overall flux density to increase but reduce the uniformity of the field direction as the flux density will be spread over a reduced volume. Once this has been accomplished, an extensive test program for evaluating the dynamic properties of MREs over a suitable 
field range will commence. The field properties for these tests will be fully specified.

\section{REFERENCES}

BOCZKOWSKA, A. \& AWIETJAN, S. 2009. Smart composites of urethane elastomers with carbonyl iron. Journal of Materials Science, 44, 4104-4111.

GORMAN, D., JERRAMS, S., EKINS, R. \& MURPHY, N. 2011. Creating a uniform magnetic field for the equi-biaxial physical testing of magnetorheological elastomers; electromagnet design, development and testing. Constitutive Models for Rubber VII. CRC Press.

GORMAN, D., JERRAMS, S., EKINS, R. \& MURPHY, N. 2012. Providing reliable data for magnetic field design in magnetorheological elastomer (MRE) damping applications. TireTechnologhy International Annual Showcase 2012, 20-22.

GRANT, I. S. \& PHILLIPS, W. R. 1975. Electromagnetism, Wiley.

HILLS, B. P., WRIGHT, K. M. \& GILLIES, D. G. 2005. A low-field, low-cost Halbach magnet array for open-access NMR. Journal of Magnetic Resonance, 175, 336-339.

MURPHY, N., HANLEY, J. \& JERRAMS, S. 2011. A method of real-time bi-axial strain control in fatigue testing of elastomers. Constitutive Models for Rubber VII. CRC Press.

STEPANOV, G. V., ABRAMCHUK, S. S., GRISHIN, D. A., NIKITIN, L. V., KRAMARENKO, E. Y. \& KHOKHLOV, A. R. 2007. Effect of a homogeneous magnetic field on the viscoelastic behavior of magnetic elastomers. Polymer, 48, 488-495.

VARGA, Z., FILIPCSEI, G. \& ZRÍNYI, M. 2006. Magnetic field sensitive functional elastomers with tuneable elastic modulus. Polymer, 47, 227-233. 